\title{
Risk factors for postoperative recurrence of ovarian endometriosis: long-term follow-up of 358 women
}

\author{
Xiao-Yan Li ${ }^{\dagger}$, Xiao-Pei Chao ${ }^{\dagger}$, Jin-Hua Leng ${ }^{*}$ (D, Wen Zhang, Jun-Ji Zhang, Yi Dai, Jing-Hua Shi, Shuang-Zheng Jia,
} Xiao-Xuan Xu, Si-Kai Chen and Yu-Shi Wu

\begin{abstract}
Objective: To explore the risk factors for the recurrence of endometrioma and the risk factors for the recurrence of endometriosis-related pain after long-term follow-up.

Methods: This study retrospectively analyzed 358 women with endometriomas who had a minimum of 5-years follow up after laparoscopic endometrioma excision, which was performed at Peking Union Medical College Hospital from January 2009 to April 2013. All women were divided into recurrence group and nonrecurrence group. Analysis was performed with regard to preoperative history, laboratory analysis, findings during surgery, and symptoms during follow-up, including improvement and recurrence.

Results: The cumulative incidence rates of recurrence from 5 to 10 years after surgery were 15.4, 16.8, 19.3, 22.5, 22.5 , and $22.5 \%$, respectively. Significant differences were found between two groups in terms of age at surgery (RR: $0.764,95 \%$ Cl: $0.615-0.949, p=0.015)$, duration of dysmenorrhea (RR: $1.120,95 \% \mathrm{Cl}: 1.054-1.190, p<0.001$ ), presence of adenomyosis (RR: 1.629, 95\% Cl: 1.008-2.630, $p=0.046$ ), CA125 level (RR: 1.856, 95\% Cl: 1.072-3.214, $p=0.021$ ) and severity of dysmenorrhea. The severity of dysmenorrhea (RR: $1.711,95 \% \mathrm{Cl}: 1.175-2.493, p=0.005$ ) and postoperative pregnancy (RR: $0.649,95 \% \mathrm{Cl}: 0.460-0.914, p=0.013$ ) were significantly correlated with endometrioma recurrence in the multivariate analysis. No significant associations were found between the recurrence rate and gravida, parity, body mass index, infertility, leiomyoma presence, the size of ovarian endometrioma, the presence of deep infiltrating endometriosis, disease stage or postoperative medication.

Conclusions: The severity of dysmenorrhea and postoperative pregnancy were independent risk factors for the recurrence of ovarian endometriomas after surgery during the long-time follow up.
\end{abstract}

Keywords: Ovarian endometriomas, Recurrence, Risk factors

\section{Introduction}

Endometriosis is a chronic benign estrogen-dependent disease. It is observed primarily in patients of reproductive age, and its prevalence in this population is estimated to be $5-10 \%$. Endometriosis is defined as the presence of active endometrial tissue outside the uterine cavity, usually on the peritoneum of the minor pelvis, ovaries and fallopian tubes and sometimes in extraperitoneal regions.

\footnotetext{
* Correspondence: lengjh_pumch@163.com

'Xiao-Yan Li and Xiao-Pei Chao contributed equally to this work.

Department of Obstetrics and Gynecology, Peking Union Medical College

Hospital, Peking Union Medical College \& Chinese Academy of Medical

Science, Shuaifuyuan No. 1, Dongcheng District, Beijing 100730, China
}

Based on the locations of the lesions, the disease is classified as peritoneal, ovarian or deep infiltrating endometriosis $[1,2]$. Endometriosis causes impaired quality of life (QoL) for women of reproductive age, and has malignant clinical manifestation despite being a benign disease. Ovarian endometriosis is the most common type, accounting $17 \%$ and $44 \%$ of all endometriosis. Laparoscopic conservative surgery has been considered the gold standard treatment for ovarian endometrioma [3, 4]. However, surgery may affect ovarian reserve function, and thus, surgery, especially repeat surgery, is not recommended for ovarian endometrioma recurrence [5-7]. The recurrence rate following surgical intervention remains high, even for

(c) The Author(s). 2019 Open Access This article is distributed under the terms of the Creative Commons Attribution 4.0 International License (http://creativecommons.org/licenses/by/4.0/), which permits unrestricted use, distribution, and 
those who receive postoperative medical therapy. Therefore, endometrioma recurrence is one of the most important unresolved problems in the management of endometriosis. The recurrence rate of ovarian endometrioma after conservative laparoscopic surgery has been reported to be $29-56 \%$ at 2 years and $43 \%$ at 5 years. When postoperative medical treatment was introduced, the endometrioma recurrence rate substantially declined to $3-11 \%$ at 2 years and $6 \%$ at 5 years $[8,9]$. A pooled analysis of 23 studies estimated recurrence rates of $21.5 \%$ at 2 years and $40.0-50.0 \%$ at 5 years after primary surgery [10]. Unfortunately, few studies have analyzed the determinants of the long-term recurrence rate for endometrioma beyond 5 years after surgery. Therefore, the aim of this study was to explore the risk factors for endometrioma recurrence and the risk factors for the recurrence of endometriosis-related pain after long-term follow up.

\section{Materials and methods}

\section{Ethical approval}

This study was approved by the Ethics Committee of Peking Union Medical College Hospital. Written informed consent was obtained from all participants.

\section{Patient population}

We collected 358 women with endometriomas who had a minimum of 5 years of postoperative follow-up after undergoing a laparoscopic cystectomy at Peking Union Medical College Hospital from January 2009 to April 2013.

The inclusion criteria were as follows: (1) the diagnosis was confirmed by pathologists; (2) ultrasonography was conducted to determine endometrioma recurrence at least 6 months after surgery; and (3) patients were observed without postoperative medications or were treated with postoperative gonadotropin-releasing hormone agonist (GnRHa) injections for 3-6 cycles, with or without a Mirena levonorgestrel-releasing intrauterine device (LNG-IUD). (4) The duration of follow-up was at least 5 years. The exclusion criteria were as follows: (1) age $<20$ or $>45$ years; (2) having underwent bilateral oophorectomy or hysterectomy; and (3) intraoperative conversion to laparotomy.

\section{Methods}

Medical charts were reviewed to collect data on age at surgery; body mass index; presence of adenomyosis or leiomyoma; surgical history; symptoms of dysmenorrhea; parity; size of endometrioma; location of cysts; serum CA125 levels; operative time; intraoperative blood loss; American Society for Reproductive Medicine (ASRM) stage [11]; postoperative medications; postoperative pregnancy; and recurrence time. The size of the ovarian endometrioma was defined as the largest diameter of cysts. Obliteration of the pouch of Douglas was defined when there was any adhesion in the pouch.

All surgeries were performed by one expert laparoscopist. In all cases, the aim of the surgical procedures was to remove all visible implants of endometriosis, complete the opening of the pouch in the operation and perform lysis of adhesion. Endometriosis was staged according to the classification of the ASRM. The presence, localization, and extent of typical powderburn and subtle lesions, adhesions, and deep infiltrating implants were recorded. Both ovaries were then completely mobilized. A sharp cortical incision was made into the cyst, and a cleavage plane was identified. A traumatic forceps and counter traction were used to strip the endometrioma from the ovarian parenchyma. Hemostasis was achieved by the selective application of bipolar coagulation. After the endometriomas were removed, all remaining visible endometriotic lesions were excised or fulgurated. Anatomical restoration was then achieved. Specimens underwent thorough histological analysis. When complications occurred, laparoscopy was converted into laparotomy. Laparotomic conversions were not included in the study.

In our center, all women are followed up according to an internal protocol. A standard gynecological examination and a transvaginal ultrasound are conducted before surgery; at 3, 6, and 12 months after surgery; and then yearly after surgery. Menstrual-reproductive factors and pain symptoms of pain are also evaluated. During the follow-up visits, patients were asked whether dysmenorrhea, pelvic pain or dyspareunia occurred during the follow-up period. The time of their first appearance and the intensity of the pain symptoms after laparoscopy were also documented. Pain was rated on the basis of a $10-\mathrm{cm}$ visual analog scale (VAS), and the intensity was divided into none (0), mild (1-4), moderate (5-7), or severe $(8-10)$. The presence of pain before surgery and pain recurrence/occurrence were defined as the recurrence of pain after a period of at least 3 months of relief after surgery. Threshold points defining different severities of pain were chosen based on a previous correlation analysis [12]. Endometrioma recurrence was determined using ultrasonography. Recurrent endometrioma was defined as the presence of a persistent ovarian cyst that had a thin wall (with a diameter of at least $2 \mathrm{~cm}$ ), regular margins, a homogenous low echogenic fluid content with scattered internal echoes and did not resolve after several successive menstrual cycles. An improvement in pain was defined as a decrease in the VAS score for at least two points. Persistent pain was defined as no improvement or an increase in the VAS score of less than 2 points after surgery. Recurrence was identified when the same VAS score as that before surgery was noted at follow-up. 


\section{Statistical analysis}

Statistical analysis was performed using the Statistics Package for Social Sciences Version 22.0 (SPSS Inc., Chicago, IL, USA). Quantitative variables were compared using t-tests and ANOVA with Bonferroni's correction for multiple testing. Fisher's exact or chi-square tests were used to analyze qualitative variables. Potential risk factors $(p<0.2)$ were identified using univariate analysis and Cox's multivariate proportional hazard analysis. The hazard ratio (HR) and 95\% confidence interval (CI) were calculated as a measure of the risk of recurrence in each study. Significance was defined as $p<0.05$.

\section{Results}

For the total of 358 women, the mean age and BMI were $33.2 \pm 5.4$ years and $21.2 \pm 2.6 \mathrm{~kg} / \mathrm{m}^{2}$. The median gravida, parity, and duration of dysmenorrhea were $1(0-10), 0(0-2)$ and 24 months (0-360 months). The median CA125 level was $54 \mathrm{U} / \mathrm{ml}(8.4-754 \mathrm{U} / \mathrm{ml})$. The mean operation time, bleeding volume and duration of follow-up were $66.4 \pm 22.3$ $\mathrm{min}, 5.4 \pm 73.5 \mathrm{ml}$, and $84.2 \pm 14.6$ months. Before surgery, dysmenorrhea was absent in $23.7 \%$ (85/358) of cases, mild in $14 \%(50 / 358)$, moderate in $21.8 \%$ (78/358), and severe in $40.5 \%(145 / 358)$. The infertility rate before surgery was $18.4 \%$ (66/358). 17.9\% (64/358) cases had concurrent leiomyoma, 53.1\% (190/358) had concurrent deep infiltrating endometriosis, and $39.9 \%$ (143/358) had concurrent adenomyosis. Abdominal and vaginal ultrasound revealed the presence of bilateral ovarian endometriomas in $46.1 \%$ $(165 / 358)$ of cases and unilateral ovarian endometriomas in 53.9\% (193/358) of cases (31.0\%, 111 left, 22.9\%, 82 right).

At laparoscopy, ovarian endometrioma was confirmed with a mean size of $5.4 \pm 2.1 \mathrm{~cm}$ on the left side and $5.3 \pm 1.9 \mathrm{~cm}$ on the right. Leiomyomas were present in $18 \%(64 / 358)$ of cases, deep infiltrating endometriosis 53.1\% (190/358), and adenomyosis 39.9\% (143/358). One hundred two patients had partial and 162 patients had total cul-de-sac obliteration. According to the rASRM classification, 134 (37.4\%) and 213 (59.5\%) cases were stage III and stage IV. Postoperative therapy was chosen on the basis of individual characteristics and careful counseling with the patient: 12 (3.4\%) women had no therapy; $12(3.4 \%)$ oral contraceptives (drospirenone and ethinylestradiol tablets for at least 1 year); 14 (3.9\%) Mirena for least 1 year, 242 (67.6\%) received GnRHa for 3-6 months, and 78 (21.8\%) had GnRHa followed by Mirena.

After more than 5-years follow-up (median 83, 60$120)$, we observed $9.4 \%(34 / 358)$ had pain recurrence, $6.4 \%(23 / 358)$ cyst recurrence and 3\% (11/358) for both. The cumulative rate of endometrioma and/or endometriosis-related pain recurrence over 5 years was $19.0 \%$. Sixty-eight women were in the recurrence group and 290 cases in the nonrecurrence group.
Dysmenorrhea recurred in 34 women (68\%), endometrioma recurred in 23 women (33.8\%), and both recurred in 11 patients (16.2\%). The clinical characteristics of the different groups of endometriomas are shown in Table 1.

Univariate analysis and Cox's multivariate proportional hazard analyses were performed. Differences were found between two groups in terms of age at surgery (RR: 0.764, 95\% CI: $0.615-0.949, p=0.015)$, duration of dysmenorrhea (RR: $1.120,95 \%$ CI 1.054-1.190, $p<0.001$ ), presence of adenomyosis (RR: $1.629,95 \%$ CI: $1.008-2.630, p=0.046$ ), CA125 level (RR: 1.856, 95\% CI: 1.072-3.214, $p=0.021$ ) and extent of dysmenorrhea. The risk ratio of mild dysmenorrhea was 4.506 (95\% CI: $1.413-14.368, p=0.011$ ), moderate was 2.451 (95\% CI: 1.433-4.193, $p=0.001$ ), and severe dysmenorrhea was 1.771 (95\% CI: 1.254-2.502, $p=0.001$ ).

No significant association was found between endometrioma and/or endometriosis-related pain recurrence rate and gravida, parity, BMI, infertility, leiomyoma presence, ovarian endometrioma size, deep infiltrating endometriosis presence, obliteration of the pouch of Douglas, disease stage and postoperative medication (Table 2). Only the extent of dysmenorrhea (RR: 1.711, 95\% CI: 1.175-2.493, $p=0.005$ ) and postoperative pregnancy (RR: 0.649 , 95\% CI: 0.460 $0.914, p=0.013$ ) were significantly correlated with endometrioma and/or endometriosis-related pain recurrence in the multivariate analysis (Table 2).

\section{Discussion}

There are a variety of factors, both clinically and surgically, that might be related to the risk of endometrioma and/or endometriosis-related pain recurrence. Accumulating evidence suggests that immune cells, adhesion molecules, extracellular matrix metalloproteinase and pro-inflammatory cytokines activate/alter peritoneal microenvironment, creating the conditions for differentiation, adhesion, proliferation and survival of ectopic endometrial cells [13-15]. In a study by Tobiume et al., the rAFS score was an independent factor associated with recurrence [16]. Chon et al. reported that dysmenorrhea and ovarian cyst separations significantly affected the postoperative recurrence rate [17]. Selcuk et al. reported that the depth of penetration of the endometrial tissue into the ovarian cyst wall was an independent risk factor for recurrence [18]. Guzel et al. reported that the CA125 level, ovarian cyst size, and history of pelvic surgery affected the recurrence rate [19]. However, it is difficult to compare the results of the above studies due to differences in the study population, the follow-up duration, and the definition of endometrioma and/or endometriosis-related pain recurrence. Notably, most of the above studies reported the endometrioma and/or endometriosis-related pain recurrence rate within 5 years after initial surgery. We 
Table 1 Baseline characteristics of the endometrioma and/or endometriosis-related pain recurrence and nonrecurrence groups

\begin{tabular}{|c|c|c|c|c|}
\hline Characteristics & Recurrence group ( $n=68$ ) & Nonrecurrence group $(n=290)$ & $x^{2}$ & $p$ value \\
\hline Age, y & $31.8 \pm 5.0$ & $32.9 \pm 5.2$ & 1.457 & 0.146 \\
\hline Gravida & $0(0-4)$ & $1(0-10)$ & 1.207 & 0.228 \\
\hline Parity & $0(0-1)$ & $0(0-2)$ & 2.385 & 0.018 \\
\hline $\mathrm{BMI}, \mathrm{kg} / \mathrm{m}^{2}$ & $20.9 \pm 2.5$ & $21.2 \pm 2.6$ & 0.814 & 0.416 \\
\hline rAFS stage & & & 3.093 & 0.377 \\
\hline Stage 1 & $0(0 \%)$ & $1(0.3 \%)$ & & \\
\hline Stage ॥ & $3(4.4 \%)$ & $7(2.4 \%)$ & & \\
\hline Stage III & $20(29.4 \%)$ & $113(39.2 \%)$ & & \\
\hline Stage IV & $45(66.2 \%)$ & $167(58.0 \%)$ & & \\
\hline \multicolumn{5}{|l|}{ Largest-diameter endometrioma, $\mathrm{cm}$} \\
\hline Left & $5.6 \pm 1.9$ & $5.4 \pm 2.2$ & -0.707 & 0.481 \\
\hline Right & $5.7 \pm 2.0$ & $5.2 \pm 1.8$ & -1.631 & 0.104 \\
\hline $\mathrm{CA} 125, \mathrm{U} / \mathrm{ml}$ & $99.23 \pm 89.72$ & $100.37 \pm 228.61$ & 0.038 & 0.970 \\
\hline Dysmenorrhea, VAS & $7(0-10)$ & $5(0-10)$ & -3.018 & 0.003 \\
\hline Extent of dysmenorrhea & & & 15.420 & 0.001 \\
\hline None & $4(5.9 \%)$ & $81(27.9 \%)$ & & \\
\hline Mild & $10(14.7 \%)$ & $40(13.8 \%)$ & & \\
\hline Moderate & $20(29.4 \%)$ & $58(20.0 \%)$ & & \\
\hline Severe & $34(50.0 \%)$ & $111(38.3 \%)$ & & \\
\hline Duration of dysmenorrhea, $\mathrm{m}$ & $84(0-360)$ & $24(0-348)$ & -3.514 & 0.001 \\
\hline Endometrioma side & & & 3.082 & 0.214 \\
\hline Left & $16(23.5 \%)$ & $95(32.8 \%)$ & & \\
\hline Right & $20(29.4 \%)$ & $62(21.4 \%)$ & & \\
\hline Bilateral & $32(47.1 \%)$ & $133(45.9 \%)$ & & \\
\hline Obliteration of cul-de-sac & & & 3.307 & 0.191 \\
\hline Absent & $13(19.1 \%)$ & $81(27.9 \%)$ & & \\
\hline Partial & $18(26.5 \%)$ & $84(29.0 \%)$ & & \\
\hline Complete & 37 (54.4\%) & $125(43.1 \%)$ & & \\
\hline Leiomyoma & $18(26.5 \%)$ & $46(16.0 \%)$ & 5.057 & $0.044(0.044)$ \\
\hline Deep infiltrating endometriosis & $41(63 \%)$ & 149 (51.4\%) & 1.758 & 0.224 \\
\hline Adenomyosis & $34(50.0 \%)$ & $108(37.4 \%)$ & 4.059 & $0.044(0.044)$ \\
\hline Postoperative dysmenorrhea relief & $55(87.3 \%)$ & $8(90.0 \%)$ & 1.770 & 0.413 \\
\hline Infertility & & & 2.408 & 0.300 \\
\hline Primary & $11(16.2 \%)$ & $32(11.0 \%)$ & & \\
\hline Secondary & $6(8.8 \%)$ & $17(5.9 \%)$ & & \\
\hline Operation & & & 0.705 & 0.590 \\
\hline Cystectomy & 65 (95.6\%) & 21 (72\%) & & \\
\hline Salpingo-oophorectomy & $3(4.4 \%)$ & $269(92.8 \%)$ & & \\
\hline Postoperative pregnancy & $24(35.3 \%)$ & $113(39.0 \%)$ & 0.611 & 0.894 \\
\hline Postoperative medication & & & 11.066 & 0.026 \\
\hline None & $0(0 \%)$ & $12(4.1 \%)$ & & \\
\hline OCP & $1(1.5 \%)$ & $11(3.8 \%)$ & & \\
\hline Mirena & $1(1.5 \%)$ & $13(4.5 \%)$ & & \\
\hline GnRHa with LNG-IUD & $9(13.2 \%)$ & $69(23.8 \%)$ & & \\
\hline
\end{tabular}


Table 1 Baseline characteristics of the endometrioma and/or endometriosis-related pain recurrence and nonrecurrence groups (Continued)

\begin{tabular}{|c|c|c|c|c|}
\hline Characteristics & Recurrence group ( $n=68$ ) & Nonrecurrence group $(n=290)$ & $x^{2}$ & $p$ value \\
\hline GnRHa without LNG-IUD & $57(83.8 \%)$ & $185(63.8 \%)$ & & \\
\hline Duration of follow-up & $83(60-120)$ & $85(60-116)$ & -0.459 & 0.646 \\
\hline
\end{tabular}

Abbreviations: BMI Body mass index, CA-125 Cancer antigen 125, cm centimeter, GnRHa Gonadotropin-releasing hormone agonist, LNG-IUD Levonorgestrelreleasing intrauterine device, $m$ month, OCP Oral contraceptive pills, VAS Visual analogue score

have little information about the recurrence rate beyond 5 years after surgery.

This study found that the extent of dysmenorrhea and postoperative pregnancy were risk factors according to the multivariate analysis. To avoid multiple collinearity, the presence of dysmenorrhea was not selected for the multivariate analysis. Several studies identified dysmenorrhea as a possible risk factor for endometrioma recurrence $[16,20]$. Our study also showed an association between the presence of dysmenorrhea after surgery and endometrioma and/or endometriosis-related pain recurrence in a univariate analysis (Fig. 1). Mechanisms of dysmenorrhea have not been uniformly determined; however, direct and indirect effects of focal bleeding from endometriotic implants, actions of inflammatory cytokines in the peritoneal cavity, and irritation or direct infiltration of nerves in the pelvic floor are causes [21]. It is hypothesized that cyclic recurrent microbleeding within endometriotic lesions with consequent inflammation may be the cause of severe dysmenorrhea among women with endometriosis [22]. Therefore, the longer the disease duration is, the deeper the lesions will infiltrate. In our series, women who suffered dysmenorrhea over 6 months had higher endometrioma and/ or endometriosis-related pain recurrence rate (Fig. 2). Over time, most patients with endometriomas had a higher rASRM score and were categorized as having advanced-stage endometriosis. In fact, most of patients (96.9\%) in this study demonstrated stage III or IV endometriosis. Adhesions may also cause deep pelvic pain associated with recurrent endometriosis, and postoperative dysmenorrhea also suggests endometrioma recurrence [23]. Because of extensive adhesions and inflammation, incomplete resection can occur in advanced stages of endometriosis. Incomplete surgical removal of endometriomas located on an endometriotic lesion or adhesion only decreases the severity of symptoms because only the removal of visualized cystic lesions or separation of adhesions is performed; Although peritoneal superficial lesions and ovarian endometriomas represent the majority of endometriotic implants within the pelvis, deep infiltrating endometriosis and extrapelvic endometriosis are the most challenging conditions to face off. Despite sometimes medical therapy is enough to reduce symptoms and, in a large number of patients a complete eradication, with nerve-sparing and vascular sparing approach is needed to restore the normal pelvic anatomy and its functions [24, 25]. In our study, all remaining visible endometriotic lesions were excised or fulgurated after the endometriomas were removed. Anatomical restoration was then achieved. However, surgery does not address the underlying mechanisms that are active and driving disease in the pelvic cavity. This naturally leads to recurrence of endometriosis in various forms after a certain period of time.

The presence of adenomyosis is another risk factor for endometrioma and/or endometriosis-related pain recurrence. It is well known that there is an overlap in the pathogenesis of endometriosis and adenomyosis [22]. Dior et al. found that sonographic features of adenomyosis may be included as a component of the clinical assessment when attempting to predict the presence of severe endometriosis [26]. We observed a statistically significant correlation between pelvic pain and the presence of adenomyosis (Fig. 3), in agreement with the previously reported results $[27,28]$. Dysmenorrhea is a risk factor in the deep adenomyotic process with a high density of endometrial glands in the myometrium [29]. Perello et al. observed a clear trend associating of the presence of dysmenorrhea with adenomyosis, without statistical significance. Among the patients reporting these symptoms, $95.5 \%$ described the intensity as severe [30]. Our study showed that the concurrence rate of adenomyosis was up to $50.0 \%$ in the recurrence group. Unresolved adenomyosis may consequently lead to pain recurrences after pelvic surgery. Therefore, correct identification of coexisting pathological conditions for DIE and adenomyosis is necessary for the development of effective surgical protocols [30].

It was reported that age at surgery is associated with the postsurgical endometrioma recurrence rate, and the reason is unclear. We postulate that endometriosis is a hormone dependent disease. Thus, the higher circulation estrogen levels in younger women may produce a more aggressive form of endometriosis; therefore, the younger, the more likely to recur [18]. Seo et al. [31] reported that, at an average follow-up time of 29 months with no postoperative medication, the cumulative endometrioma recurrence rates were $43.3 \%$ for patients aged 20-29 
Table 2 Univariate and multivariate analysis of risk factors in the endometrioma and/or endometriosis-related pain recurrence and nonrecurrence groups

\begin{tabular}{|c|c|c|c|c|c|c|}
\hline \multirow[t]{2}{*}{ Factor } & \multicolumn{3}{|c|}{ Univariate analysis } & \multicolumn{3}{|c|}{ Multivariate analysis } \\
\hline & Relative risk & $95 \% \mathrm{Cl}$ & $P$ value & Relative risk & $95 \% \mathrm{Cl}$ & $P$ value \\
\hline Age at surgery (per 5 years) & 0.764 & $0.615-0.949$ & 0.015 & 0.738 & $0.520-1.048$ & 0.090 \\
\hline \multicolumn{7}{|l|}{ Gravida } \\
\hline 0 & 1.000 & & & & & \\
\hline 1 & 0.673 & $0.370-1.224$ & 0.194 & & & \\
\hline 2 (and above) & 0.757 & $0.557-1.029$ & 0.075 & & & \\
\hline \multicolumn{7}{|l|}{ Parity } \\
\hline 0 & 1.000 & & & & & \\
\hline 1 & 0.478 & $0.261-0.876$ & 0.017 & 0.002 & $0.000-1.222$ & 0.974 \\
\hline 2 & 0.216 & $0.011-4.254$ & 0.314 & & & \\
\hline BMI & 0.820 & $0.478-1.407$ & 0.470 & & & \\
\hline Infertility & 1.527 & $0.882-2.644$ & 0.131 & & & \\
\hline Extent of dysmenorrhea & & & & 1.711 & $1.175-2.493$ & 0.005 \\
\hline None & 1.000 & & & & & \\
\hline Mild & 4.506 & $1.413-14.368$ & 0.011 & & & \\
\hline Moderate & 2.451 & $1.433-4.193$ & 0.001 & & & \\
\hline Severe & 1.771 & $1.254-2.502$ & 0.001 & & & \\
\hline Duration of dysmenorrhea (per year) & 1.120 & $1.054-1.190$ & $<0.001$ & 1.026 & $0.944-1.114$ & 0.548 \\
\hline Extent of dysmenorrhea & 2.534 & $1.408-4.563$ & 0.002 & & & \\
\hline \multicolumn{7}{|l|}{ VAS (0-4) } \\
\hline \multicolumn{7}{|l|}{ VAS (5-10) } \\
\hline Dyspareunia & 1.734 & $1.049-2.866$ & 0.032 & 1.081 & $0.511-2.285$ & 0.839 \\
\hline Chronic pelvic pain & 0.959 & $0.503-1.828$ & 0.898 & & & \\
\hline Dyschezia & 0.735 & $0.268-2.018$ & 0.550 & & & \\
\hline Presence of leiomyoma & 1.661 & $0.969-2.848$ & 0.065 & 1.713 & $0.825-3.555$ & 0.149 \\
\hline Presence of adenomyosis & 1.629 & $1.008-2.630$ & 0.046 & 1.446 & $0.719-2.908$ & 0.301 \\
\hline Presence of deep infiltrating endometriosis & 1.380 & $0.849-2.243$ & 0.194 & 1.118 & $0.582-2.149$ & 0.737 \\
\hline CA125 level (45 U/ml) & 1.856 & $1.072-3.214$ & 0.021 & 1.020 & $0.965-1.078$ & 0.491 \\
\hline rARSM stage & 1.204 & $0.774-1.873$ & 0.409 & & & \\
\hline Bilateral involvement & 1.046 & $0.650-1.684$ & 0.853 & & & \\
\hline Large dimeter (per 10 mm) & 1.016 & $0.944-1.093$ & 0.674 & & & \\
\hline \multicolumn{7}{|l|}{ Postoperative medication } \\
\hline None & 1.000 & & & & & \\
\hline GnRHa & 21.818 & $0.125-3810.382$ & 0.242 & & & \\
\hline OCP & 4.057 & 0.019-856.392 & 0.610 & & & \\
\hline Mirena & 2.741 & $0.048-155.496$ & 0.624 & & & \\
\hline GnRHa+Mirena & 1.991 & $0.375-9.730$ & 0.436 & & & \\
\hline Postoperative pregnancy & 0.938 & $0.655-1.344$ & 0.728 & 0.649 & $0.460-0.914$ & 0.013 \\
\hline
\end{tabular}

Abbreviations: BMI Body mass index, CA-125 Cancer antigen 125, GnRHa Gonadotropin-releasing hormone agonist, OCP Oral contraceptive pills, VAS Visual analogue score

years, $22.5 \%$ for $30-39$ years, and $10.2 \%$ for $40-45$ years. In our study, the cumulative endometrioma and/or endometriosis-related pain recurrence rates were $24.2 \%$ for patients aged $20-30$ years, $17.7 \%$ for $31-40$ years, and $7.9 \%$ for $41-45$ years, which was consistent with the literature. Our study also found that 33.5 years is a cutoff value in the ROC age analysis (Fig. 4a). Patients who are younger than 33.5 years may have a higher risk for 


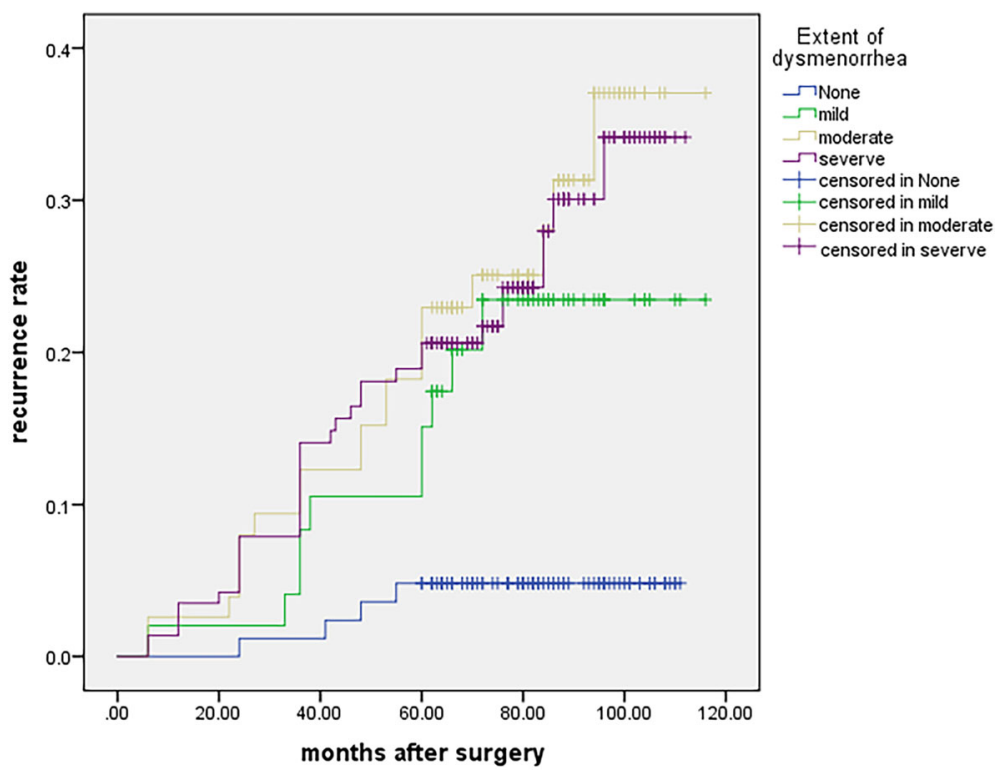

Fig. 1 Kaplan-Meier curves presenting the cumulative rate of recurrence according to the severity of dysmenorrhea. There were significant differences between the four groups according to the log-rank test analysis $\left(X^{2}=11.487, p=0.001\right)$

recurrence (Fig. 4b). Our previous study showed that laparoscopic cystectomy for endometrioma with an age greater than 35 years may decrease the remaining ovarian reserve [32]. Therefore, a decrease in the rate of endometrioma and/or endometriosis-related pain recurrence also results in a reduction in ovarian reserve function. 33 to 35 years of age is a "dilemma window", and we should pay more attention to patients in this age group when performing surgery.
Preventive use of medications after operation is recommended for patients with a high risk of recurrence [33-36]. Many studies have investigated factors determining the recurrence of endometrioma and pain after surgery $[16,19,20]$. The mechanism of oral contraceptive pills (OCPs) reducing ovarian endometrioma recurrence is unclear. OCP increases apoptosis and decreases cell proliferation in eutopic endometria, which can decrease both recurrence from small
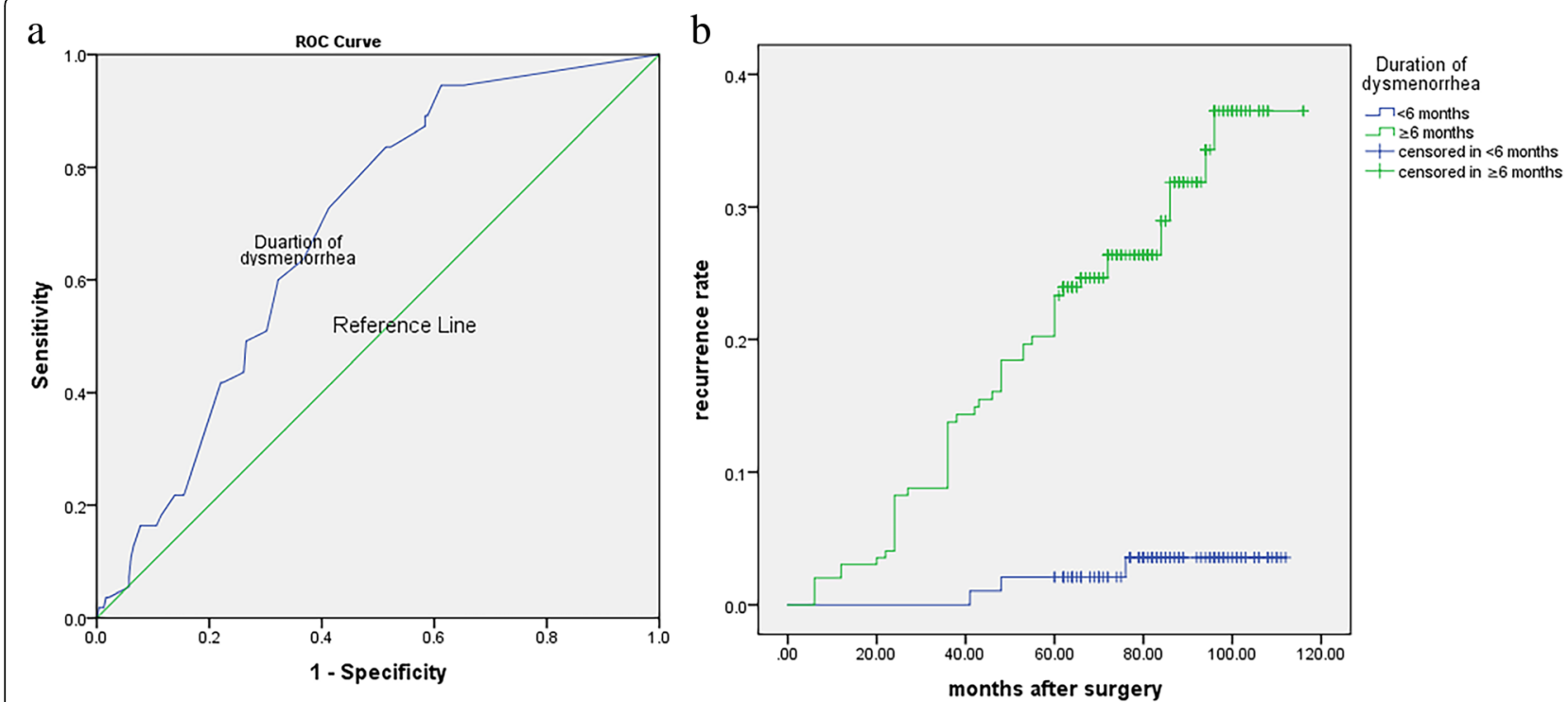

Fig. 2 a The ROC analysis of duration of dysmenorrhea in recurrent endometrioma patients. Area under curve: 0.689, Cut-off value: 5.5 months; b Kaplan-Meier curves presenting the cumulative rate of recurrence according to duration of dysmenorrhea ( $<6$ months or $\geq 6$ months). There were significant differences between the two groups according to the log-rank test analysis $\left(x^{2}=22.352, p<0.001\right)$ 


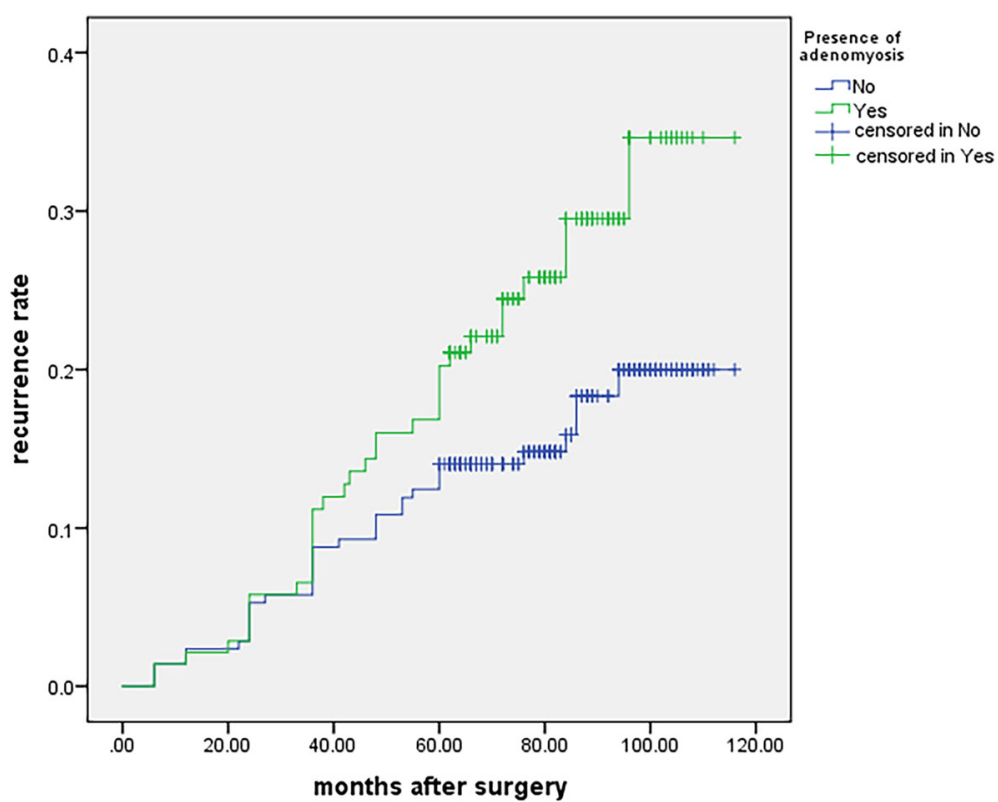

Fig. 3 Kaplan-Meier curves presenting the cumulative rate of recurrence according to the presence of adenomyosis. There were significant differences between the two groups according to the log-rank test analysis $\left(x^{2}=4.113, p=0.043\right)$

endometriotic foci not seen at surgery and de novo disease development [33]. Some reports suggest that ovarian endometrioma can develop from ovarian follicles or the corpus luteum and that consequent inhibition of ovulation may decrease the risk of endometrioma development [36-38]. Regardless of the mechanism, the present and previous studies suggest that postoperative medical treatment is known to delay but not completely prevent recurrence. Vercellini et al. [39] reported that postoperative use of GnRHa could only prolong the recurrence interval but could not improve the overall recurrence rate. There is no consensus regarding whether the LNGIUD could reduce the endometrioma recurrence rate either [40]. Notably, Jee et al. [41] reported that although postoperative GnRH agonist treatment does
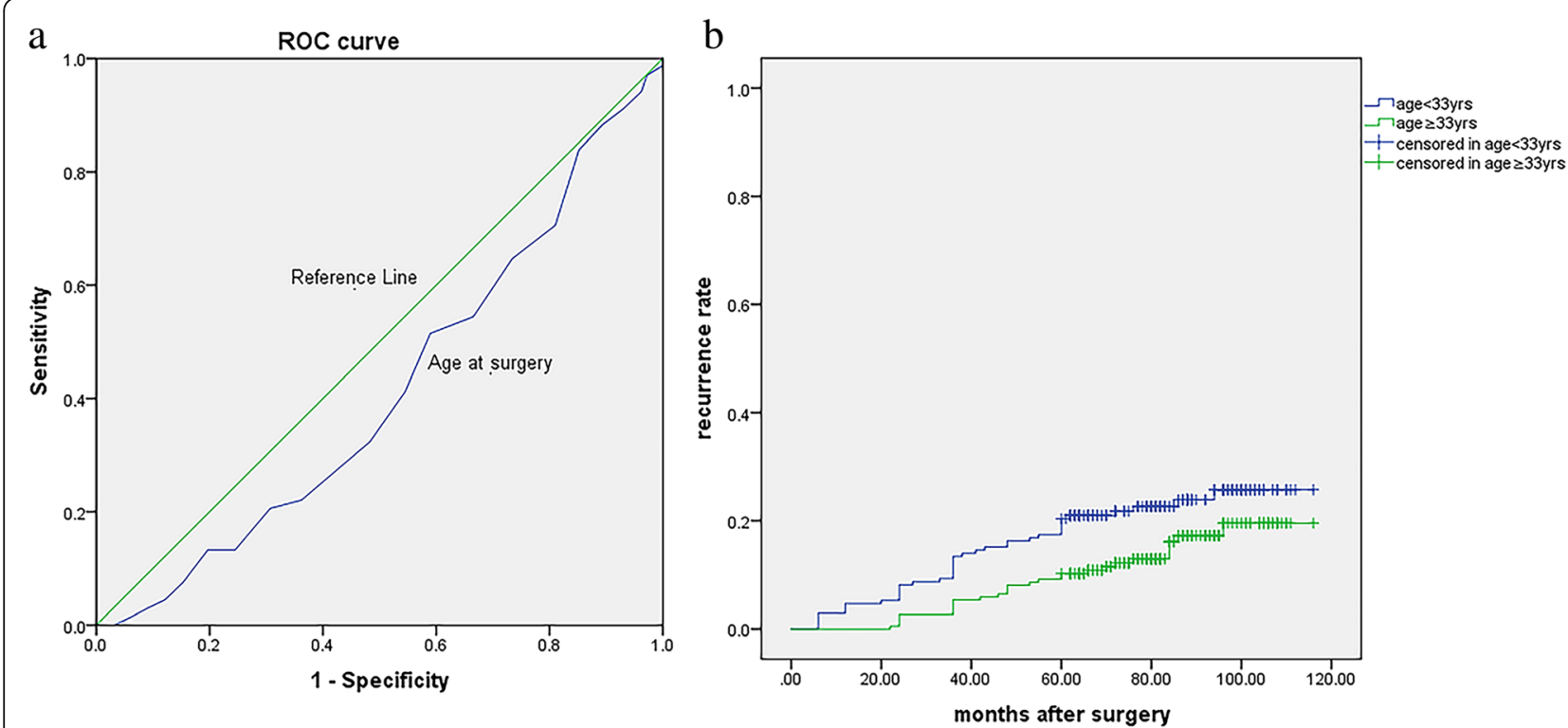

Fig. 4 a The ROC analysis of age at surgery in recurrent endometrioma patients. Area under curve: 0.413, Cut-off value: 33.5 yrs.; b Kaplan-Meier curves presenting the cumulative rate of recurrence according to age at surgery ( $<33 \mathrm{yrs}$. or $\geq 33 \mathrm{yrs}$ ). There were significant differences between the two groups according to the log-rank test analysis $\left(x^{2}=22.352, p<0.001\right)$ 
not reduce objective disease recurrence in stage III/IV disease, GnRHa delays the time of recurrence, as indicated by Vercellini et al. [39]. In our study, we also failed to observe a benefit for postoperative medication in preventing endometrioma and/or endometriosis-related pain recurrence. Patients with advanced-stage endometriosis may prefer to take postoperative medication. This may partially explain why a statistically significant difference was not reached in terms of postoperative medication between patients with and without recurrence. We found that the estimated cumulative endometrioma and/or endometriosis-related pain recurrence rates at 1 to 10 years after surgery were $2.2,5.3$, $9.2,12.0,15.4,16.8,19.3,22.5,22.5$, and $22.5 \%$, respectively. These relatively low endometrioma and/or endometriosisrelated pain recurrence rates may be due to most patients having postoperative medication applied in our study. The recurrent rate increased yearly until 8 years after surgery.

Most ovarian endometriomas are composed of an extraovarian pseudocystic structure with no cystic wall but are surrounded by fibrosis with underlying ovarian cortical follicles [42]. Therefore, the inner surface of an endometrioma is lined by endometriosis with variable penetration into the surrounding fibrosis. The mean cyst wall thickness varied between 1.2 and $1.6 \mathrm{~mm}$. Endometriosis tissue covers the inner aspect of the cyst for approximately $60 \%$ of its surface with a mean depth of penetration of $0.6 \mathrm{~mm}$ [43]. With the aging of the endometrioma, infiltration and invasion of the ovarian interstitial, resulting in the incomplete removal of endometriomas [42]. Concordantly, the univariate analysis in our study shows that the duration of dysmenorrhea is significantly associated with endometrioma and/ or endometriosis-related pain recurrence (Fig. 2).

It is clear that the prevalence of recurrent endometrioma varies according to whether there is a successful pregnancy after surgical treatment of the endometrioma $[44,45]$. Koga et al. demonstrated that patients with postoperative pregnancies had much lower rates of recurrence, which indicates that a subsequent pregnancy may have a protective effect on endometrioma recurrence [46]. In our study, postoperative pregnancies also prevented endometrioma recurrence (OR: $0.649,95 \% \mathrm{CI}: 0.460-0.914, p=0.013$ ).

The merits of our study were the long time follow-up of more than 5 years, all with detailed record clinical data, the same experienced surgeon, and the large sample size. However, there are some limitations. This was a retrospective study, and it may contain biases with regard to patient characteristics; women with severe forms of endometriosis may prefer to receive preoperative or postoperative medical treatment, and this is a single center study. Recurrent endometrioma was defined by ultrasound as the presence of a persistent ovarian cyst and did not resolve after several successive menstrual cycles. It depends mainly on the skill and experience of radiologists. These limitations may have resulted in an under or over estimation of the associations in our study.

In conclusion, we conducted a long-term follow-up study for more than 5 years. The rate of endometrioma and/or endometriosis-related pain recurrence increased yearly in the first 8 years. Our research revealed that the extent of dysmenorrhea and postoperative pregnancy are independent risk factors for endometrioma and/or endometriosis-related pain recurrence. For patients who have serious dysmenorrhea, we should pay more attention to their risk for endometrioma and/or endometriosisrelated pain recurrence and apply individual management to achieve better efficacy.

\section{Acknowledgments \\ We appreciate the stuff at Peking Union Medical College Hospital for their diligent clinical work and precise data recording about the cases we reported in this article.}

\section{Authors'contributions}

$X Y-L$ and $J H-L$ developed the idea for the project. The study was designed by XY-L and XP-C, and JJ-Z, Y-D, JH-S, SZ-J, and WZ performed the data analysis and takes full responsibility for the integrity of the data. XX-X, YS-W, $W Z$ and $X P-C$ drafted the manuscript with inputs and critical discussion from $J H-L$ and $X Y-L$. The final version has been approved by all authors.

\section{Funding}

Our work was supported by the National Key R\&D Program of China (No. 2017YFC1001200), National Natural Science Foundation of China (No. 81501237), and 2016 PUMCH Science Fund for Junior Faculty (No. PUMCH-2016-2.2).

\section{Availability of data and materials}

The dataset supporting the conclusions of this article is included within the article and its additional files.

Ethics approval and consent to participate

This study was approved by the Ethics Committee of Peking Union Medical College Hospital.

\section{Consent for publication}

Not applicable.

\section{Competing interests}

The authors declare that they have no competing interests.

Received: 11 April 2019 Accepted: 13 August 2019

Published online: 30 August 2019

\section{References}

1. Chapron C, Vercellini P, Barakat H, Vieira M, Dubuisson JB. Management of ovarian endometriomas. Hum Reprod Update. 2002;8(6):591-7.

2. Muzii L, Di Tucci C, Di Mascio D, Di Feliciantonio M, Capri O, Pietrangeli D, et al. Current management of ovarian endometriomas. Minerva Ginecol. 2018;70(3):286-94

3. Luu TH, Uy-Kroh MJ. New developments in surgery for endometriosis and pelvic pain. Clin Obstet Gynecol. 2017;60(2):245-51.

4. Deckers P, Ribeiro SC, Simoes RDS, Miyahara C, Baracat EC. Systematic review and meta-analysis of the effect of bipolar electrocoagulation during laparoscopic ovarian endometrioma stripping on ovarian reserve. Int J Gynaecol Obstet. 2018;140(1):11-7.

5. Mehdizadeh Kashi A, Chaichian S, Ariana S, Fazaeli M, Moradi Y, Rashidi M, et al. The impact of laparoscopic cystectomy on ovarian reserve in patients with unilateral and bilateral endometrioma. Int J Gynaecol Obstet. 2017;136(2):200-4.

6. Ozaki R, Kumakiri J, Tinelli A, Grimbizis GF, Kitade M, Takeda S. Evaluation of factors predicting diminished ovarian reserve before and after laparoscopic cystectomy for ovarian endometriomas: a prospective cohort study. J Ovarian Res. 2016;9(1):37. 
7. Muzii L, Achilli C, Lecce F, Bianchi A, Franceschetti S, Marchetti C, et al. Second surgery for recurrent endometriomas is more harmful to healthy ovarian tissue and ovarian reserve than first surgery. Fertil Steril. 2015;103(3):738-43.

8. Lee DY, Bae DS, Yoon BK, Choi D. Post-operative cyclic oral contraceptive use after gonadotrophin-releasing hormone agonist treatment effectively prevents endometrioma recurrence. Hum Reprod. 2010;25(12):3050-4.

9. Seracchioli R, Mabrouk M, Frasca C, Manuzzi L, Montanari G, Keramyda A, et al. Long-term cyclic and continuous oral contraceptive therapy and endometrioma recurrence: a randomized controlled trial. Fertil Steril. 2010;93(1):52-6.

10. Guo SW. Recurrence of endometriosis and its control. Hum Reprod Update. 2009;15(4):441-61.

11. Revised American Society for Reproductive Medicine classification of endometriosis: 1996. Fertil Steril 1997; 67(5):817-821.

12. Porpora MG, Pallante D, Ferro A, Crisafi B, Bellati F, Benedetti Panici P. Pain and ovarian endometrioma recurrence after laparoscopic treatment of endometriosis: a long-term prospective study. Fertil Steril. 2010;93(3):716-21.

13. Lagana AS, Vitale SG, Salmeri FM, Triolo O, Ban Frangez H, Vrtacnik-Bokal E, et al. Unus pro omnibus, omnes pro uno: a novel, evidence-based, unifying theory for the pathogenesis of endometriosis. Med Hypotheses. 2017;103:10-20.

14. Vitale SG, Capriglione S, Peterlunger I, La Rosa VL, Vitagliano A, Noventa M, et al. The role of oxidative stress and membrane transport systems during endometriosis: a fresh look at a busy corner. Oxidative Med Cell Longev. 2018;2018:7924021.

15. Lagana AS, Garzon S, Franchi M, Casarin J, Gullo G, Ghezzi F. Translational animal models for endometriosis research: a long and windy road. Ann Transl Med. 2018;6(22):431

16. Tobiume T, Kotani Y, Takaya H, Nakai H, Tsuji I, Suzuki A, et al. Determinant factors of postoperative recurrence of endometriosis: difference between endometrioma and pain. Eur J Obstet Gynecol Reprod Biol. 2016:205:54-9.

17. Chon SJ, Lee SH, Choi JH, Lee JS. Preoperative risk factors in recurrent endometrioma after primary conservative surgery. Obstet Gynecol Sci. 2016; 59(4):286-94.

18. Selcuk S, Cam C, Koc N, Kucukbas M, Ozkaya E, Eser A, et al. Evaluation of risk factors for the recurrence of ovarian endometriomas. Eur J Obstet Gynecol Reprod Biol. 2016:203:56-60.

19. Guzel Al, Topcu HO, Ekilinc S, Tokmak A, Kokanali MK, Cavkaytar S, et al. Recurrence factors in women underwent laparoscopic surgery for endometrioma. Minerva Chir. 2014;69(5):277-82.

20. Han S, Lee H, Kim S, Joo J, Suh D, Kim K, et al. Risk factors related to the recurrence of endometrioma in patients with long-term postoperative medical therapy. Ginekol Pol. 2018;89(11):611-7.

21. Lee DY, Kim HJ, Yoon BK, Choi D. Factors associated with the laterality of recurrent endometriomas after conservative surgery. Gynecol Endocrinol. 2013:29(11):978-81.

22. Fauconnier A, Chapron C. Endometriosis and pelvic pain: epidemiological evidence of the relationship and implications. Hum Reprod Update. 2005; 11(6):595-606

23. Kucukbas M, Kurek Eken M, Ilhan G, Senol T, Herkiloglu D, Kapudere B. Which factors are associated with the recurrence of endometrioma after cystectomy? J Obstet Gynaecol. 2018;38(3):372-6.

24. Lagana AS, Vitale SG, Trovato MA, Palmara VI, Rapisarda AM, Granese R, et al. Full-thickness excision versus shaving by laparoscopy for intestinal deep infiltrating endometriosis: rationale and potential treatment options. Biomed Res Int. 2016;2016:3617179.

25. Raffaelli R, Garzon S, Baggio S, Genna M, Pomini P, Lagana AS, et al. Mesenteric vascular and nerve sparing surgery in laparoscopic segmental intestinal resection for deep infiltrating endometriosis. Eur J Obstet Gynecol Reprod Biol. 2018:231:214-9.

26. Dior UP, Nisbet D, Fung JN, et al. The Association of Sonographic Evidence of Adenomyosis with Severe Endometriosis and Gene Expression in Eutopic Endometrium. J Minim Invasive Gynecol. 2019;26(5):941-8.

27. Genc M, Genc B, Cengiz H. Adenomyosis and accompanying gynecological pathologies. Arch Gynecol Obstet. 2015;291(4):877-81.

28. Aleksandrovych V, Basta P, Gil K. Current facts constituting an understanding of the nature of adenomyosis. Adv Clin Exp Med. 2019;28(6):839-46.

29. Mehasseb MK, Bell SC, Pringle JH, Habiba MA. Uterine adenomyosis is associated with ultrastructural features of altered contractility in the inner myometrium. Fertil Steril. 2010;93(7):2130-6.

30. Perello MF, Martinez-Zamora MA, Torres X, Munros J, Balasch Cortina J, Carmona F. Endometriotic pain is associated with Adenomyosis but not with the compartments affected by deep infiltrating endometriosis. Gynecol Obstet Investig. 2017:82(3):240-6.

31. Seo JW, Lee DY, Yoon BK, Choi D. The age-related recurrence of endometrioma after conservative surgery. Eur J Obstet Gynecol Reprod Biol. 2017;208:81-5.

32. Shi J, Leng J, Cui Q, Lang J. Follicle loss after laparoscopic treatment of ovarian endometriotic cysts. Int J Gynaecol Obstet. 2011;115(3):277-81.

33. Vercellini $P$, Somigliana E, Daguati R, Vigano P, Meroni F, Crosignani PG. Postoperative oral contraceptive exposure and risk of endometrioma recurrence. Am J Obstet Gynecol. 2008;198(5):504 e1-5.

34. Zhu S, Zhu Y, Liu Y, Zhang H. Comparison of outcomes of different postoperative hormone therapy in the treatment of ovarian endometriosis: a brief report. Adv Ther. 2018;35(6):857-63.

35. Reis FM, Petraglia F, Kim MK. Postoperative Levonorgestrel-releasing intrauterine system insertion after gonadotropin-releasing hormone agonist treatment for preventing Endometriotic cyst recurrence: a prospective observational study. F1000Research. 2018;25(1):39-43.

36. Seo JW, Lee DY, Yoon BK, Choi D. The efficacy of postoperative cyclic Oral contraceptives after gonadotropin-releasing hormone agonist therapy to prevent Endometrioma recurrence in adolescents. J Pediatr Adolesc Gynecol. 2017;30(2):223-7.

37. Takamura M, Koga K, Osuga $Y$, Takemura $Y$, Hamasaki $K$, Hirota $Y$, et al. Post-operative oral contraceptive use reduces the risk of ovarian endometrioma recurrence after laparoscopic excision. Hum Reprod. 2009;24(12):3042-8

38. Muzii L, Di Tucci C, Achilli C, Di Donato V, Musella A, Palaia l, et al. Continuous versus cyclic oral contraceptives after laparoscopic excision of ovarian endometriomas: a systematic review and metaanalysis. Am J Obstet Gynecol. 2016;214(2):203-11.

39. Vercellini P, Crosignani PG, Fadini R, Radici E, Belloni C, Sismondi P. A gonadotrophin-releasing hormone agonist compared with expectant management after conservative surgery for symptomatic endometriosis. $\mathrm{Br}$ J Obstet Gynaecol. 1999;106(7):672-7.

40. Kim MK, Chon SJ, Lee JH, Yun BH, Cho S, Choi YS, et al. Postoperative Levonorgestrel-releasing intrauterine system insertion after gonadotropin-releasing hormone agonist treatment for preventing Endometriotic cyst recurrence: a prospective observational study. Reprod Sci (Thousand Oaks, Calif). 2018;25(1):39-43.

41. Jee BC, Lee JY, Suh CS, Kim SH, Choi YM, Moon SY. Impact of GnRH agonist treatment on recurrence of ovarian endometriomas after conservative laparoscopic surgery. Fertil Steril. 2009;91(1):40-5.

42. Gordts S, Campo R. Modern approaches to surgical management of endometrioma. Best Pract Res Clin Obstet Gynaecol. 2019.

43. Muzii L, Bianchi A, Bellati F, Cristi E, Pernice M, Zullo MA, et al. Histologic analysis of endometriomas: what the surgeon needs to know. Fertil Steril. 2007:87(2):362-6

44. Liu X, Yuan L, Shen F, Zhu Z, Jiang H, Guo SW. Patterns of and risk factors for recurrence in women with ovarian endometriomas. Obstet Gynecol. 2007;109(6):1411-20.

45. Ashrafi M, Sadatmahalleh SJ, Akhoond MR, Talebi M. Evaluation of risk factors associated with endometriosis in infertile women. Int J Fertil Steril. 2016;10(1):11-21.

46. Koga K, Takemura Y, Osuga Y, Yoshino O, Hirota Y, Hirata T, et al. Recurrence of ovarian endometrioma after laparoscopic excision. Hum Reprod. 2006; 21(8):2171-4.

\section{Publisher's Note}

Springer Nature remains neutral with regard to jurisdictional claims in published maps and institutional affiliations. 